\title{
Selective separation of manganese, cobalt and nickel in a fully aqueous system
}

Nicolas Schaeffer, ${ }^{[a]}$ Helena Passos, ${ }^{[a]}$ Matthieu Gras, ${ }^{[b]}$ Sílvia Juliana Rodriguez Vargas, ${ }^{[a]}$ Márcia C. Neves, ${ }^{[a]}$ Lenka Svecova, ${ }^{[b]}$ Nicolas Papaiconomou ${ }^{[c]}$ and João A.P. Coutinho*[a]

[a] CICECO, Aveiro Institute of Materials, Department of Chemistry, University of Aveiro, Campus Universitário de Santiago, 3810-193 Aveiro, Portugal.

[b] Univ. Grenoble Alpes, Univ. Savoie Mont Blanc, CNRS, Grenoble INP, LEPMI, 38000 Grenoble, France.

[c] Université Nice Sophia Antipolis, Department of Chemistry, 28 Avenue Valrose, 06103 Nice, France

* Corresponding authors: jcoutinho@ua.pt

\section{Number of pages: 13}

Number of Figures: 9

Number of Tables: 3 


\section{METHODOLOGY}

\section{Materials and Methods}

Metal concentration was determined by atomic adsorption spectroscopy (AAS) using a PinAAcle 900F (Perkin Elmer) spectrometer. ${ }^{1} \mathrm{H}-{ }^{12} \mathrm{C}$ - and ${ }^{31} \mathrm{P}-\mathrm{NMR}$ spectroscopy of $\left[\mathrm{P}_{44414}\right] \mathrm{Cl}$ solutions were recorded using a $300 \mathrm{MHz}$ Bruker Avance III spectrometer with $\mathrm{D}_{2} \mathrm{O}$ as solvent. $\left[\mathrm{P}_{44414}\right]^{+}$concentration in the was determined by quantitative ${ }^{1} \mathrm{H}-\mathrm{NMR}$ with benzene as internal standard. Water content of the IL-rich phase was determined using an $831 \mathrm{KF}$ Coulometer (Metrohm). Sample morphology was analysed by scanning electron microscopy (SEM) with energy-dispersive X-ray spectroscopy (EDS) using a Bruker Nano $\mathrm{GmbH}$ microscope after carbon sputter-coating. Fourier transform infrared (FTIR) spectra were recorded on a Galaxy Series 7000 spectrometer equipped with an attenuated total reflectance (ATR) accessory (Golden Gate). Temperature controlled UV-vis spectra were recorded on a Shimadzu UV-1800 spectrophotometer fitted with a TCC-100 Thermoelectrically cell holder (accuracy of $\pm 0.1{ }^{\circ} \mathrm{C}$ ). Dynamic Light Scattering (DLS) measurements (Malvern Zetasizer Nano-ZS) were carried out to evaluate the change in aggregate size with temperature. Samples were irradiated with red light (a HeNe laser, a wavelength of $565 \mathrm{~nm}$ ) and the intensity fluctuations of the scattered light were detected at a backscattering angle of $173^{\circ}$ to generate an autocorrelation function. The cumulant analysis of this function provided by software DTS v 7.03 yielded the particle size and the distribution width. All studied system compositions were determined by the weight quantification of all components added within an uncertainty of $\pm 10^{-3} \mathrm{~g}$ using a Mettler Toledo XP205 analytic balance.

The IL tributyltetradecyl phosphonium chloride $\left(\left[\mathrm{P}_{44414}\right] \mathrm{Cl}\right)$ was purchased from Iolitec in $95 \mathrm{wt} \%$ purity and confirmed as 97.1 wt.\% pure by quantitative ${ }^{1} \mathrm{H}-\mathrm{NMR}$ analysis. Cobalt chloride hexahydrate $(99 \%$ purity) and manganese chloride tetrahydrate (99\% purity) were obtained from Merck and nickel chloride hexahydrate (97\% purity) was acquired from BDH Chemicals. Hydrochloric acid (37\%) was obtained from Fisher Scientific and anhydrous sodium carbonate (99.9\% purity) from Prolabo. Ultrapure, double distilled water passed through a reverse osmosis system and further treated with a Milli-Q plus 185 water purification apparatus $(18.2 \mathrm{~m} \Omega$ at $298 \mathrm{~K})$ was used for all experiments.

\section{Calibration of ozone generator}

A commercially available domestic ozone generator (Lufthous $\mathrm{O}_{3}$ from Lufthous) was used in all experiments delivering a fixed gas flow rate of $1.0 \mathrm{~L} \cdot \mathrm{min}^{-1}$ monitored using a flow meter. The ozone flow rate was determined by colorimetric titration using potassium iodide and sodium thiosulfate $\left(\mathrm{Na}_{2} \mathrm{~S}_{2} \mathrm{O}_{3}\right)$. Firstly, in a cylindrical vessel of diameter $5 \mathrm{~cm}$ and height $30 \mathrm{~cm}$, ozone was bubbled through a $100 \mathrm{~mL}$ 
solution of KI of know molarity for a determined time resulting in an intense orange coloured final solution according to the reaction:

$2 \mathrm{KI}$ (colourless) $+\mathrm{O}_{3}+\mathrm{H}_{2} \mathrm{O} \rightarrow \mathrm{I}_{2}$ (orange) $+2 \mathrm{KOH}+\mathrm{O}_{2}$

Secondly, after 10 min of oxidation the obtained orange solution was titrated using a $\mathrm{Na}_{2} \mathrm{~S}_{2} \mathrm{O}_{3}$ solution of known molarity until colourless according to the reaction:

$\mathrm{I}_{2}$ (orange) $+2 \mathrm{Na}_{2} \mathrm{~S}_{2} \mathrm{O}_{3} \rightarrow 2 \mathrm{NaI}$ (colourless) $+\mathrm{Na}_{2} \mathrm{~S}_{4} \mathrm{O}_{6}$

This procedure was repeated twice and a consistent ozone flow rate of $0.768 \mathrm{~g} \cdot \mathrm{hr}^{-1}\left(0.016 \mathrm{~mol}^{\left.-\mathrm{hr}^{-1}\right)}\right.$ was obtained.

\section{Determination of $\mathrm{MCl}_{2}-\left[\mathrm{P}_{44414}\right] \mathrm{Cl}-\mathrm{H}_{2} \mathrm{O}$ phase diagram and cobalt speciation with temperature}

The procedure followed in this work for the experimental determination of binodal curves for ternary and quaternary systems was previously reported. ${ }^{1}$ The binodal curves of the $\mathrm{NiCl}_{2}-\left[\mathrm{P}_{44414}\right] \mathrm{Cl}-\mathrm{H}_{2} \mathrm{O}$ ternary system and $\mathrm{NiCl}_{2}-\left[\mathrm{P}_{44414}\right] \mathrm{Cl}-\mathrm{HCl}-\mathrm{H}_{2} \mathrm{O}$ quaternary system at $25.0{ }^{\circ} \mathrm{C}$ and/or $50.0{ }^{\circ} \mathrm{C}$ were determined by the cloud point titration method in a temperature-controlled cell under agitation and atmospheric pressure. Starting aqueous solutions of $\left[\mathrm{P}_{44414}\right] \mathrm{Cl}(70.0 \mathrm{wt} \%)$ and saturated solutions of chloride salts were prepared and used for the determination of the binodal curves. The phase diagrams for the quaternary $\mathrm{NiCl}_{2}-$ $\left[\mathrm{P}_{44414}\right] \mathrm{Cl}-\mathrm{HCl}-\mathrm{H}_{2} \mathrm{O}$ system containing a fixed $\mathrm{HCl}$ concentration $(3.7 \mathrm{wt} \%)$ was obtained by maintaining a constant $3.7 \mathrm{wt} . \% \mathrm{HCl}$ concentration in all solutions used throughout the cloud point titration procedure. The ternary and quaternary system compositions were determined by the weight quantification of all components added within an uncertainty of $\pm 10^{-3} \mathrm{~g}$.

\section{REFERENCES}

[1] Neves, C.M.S.S.; Ventura, S.P.M.; Freire, M.G.; Marrucho, I.M.; Coutinho, J.A.P. Evaluation of Cation Influence on the Formation and Extraction Capability of Ionic-Liquid-Based Aqueous Biphasic Systems. J. Phys. Chem. B, 2009, 113, 5194-5199. 


\section{FIGURES}

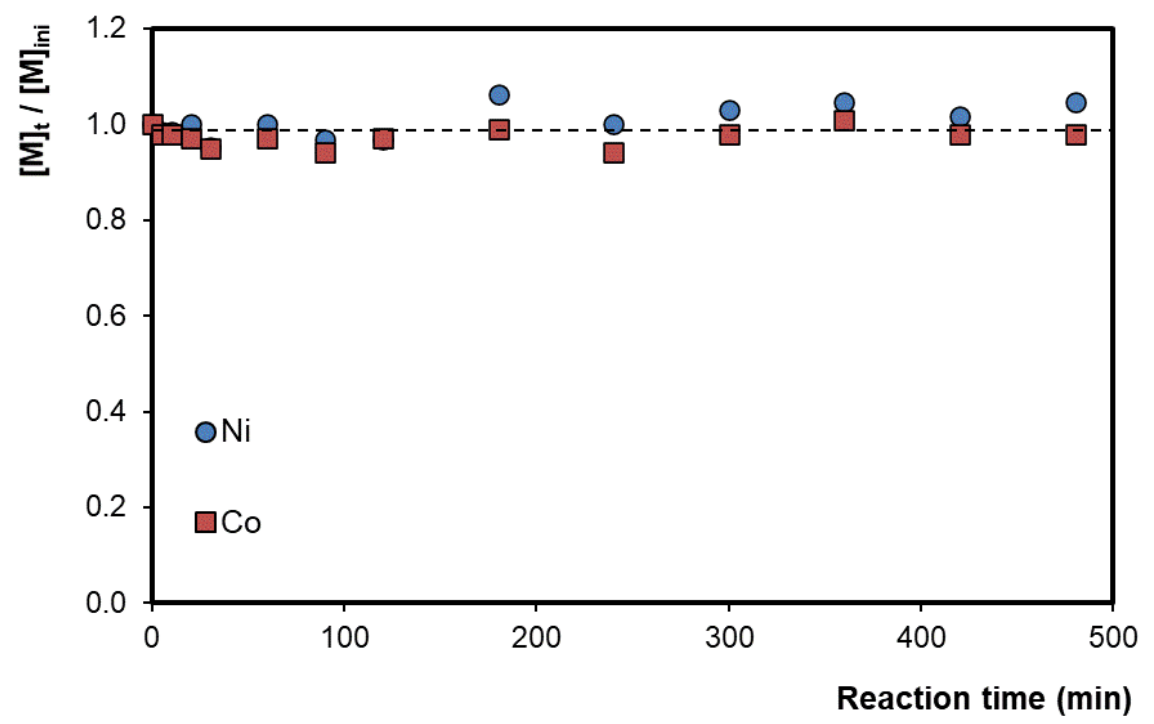

Figure S1. Evolution of the cobalt and nickel concentration with time during the continued ozonation of manganese in aqueous solution. The concentration of cobalt and nickel are standardized according to their initial concentration (Mn:Co:Ni molar ratio of 0.07:0.17:1.00 and $\left.\left[\mathrm{NiCl}_{2} \cdot 6 \mathrm{H}_{2} \mathrm{O}\right]_{\text {ini }}=0.32 \mathrm{~mol} . \mathrm{kg}^{-1}\right)$. 


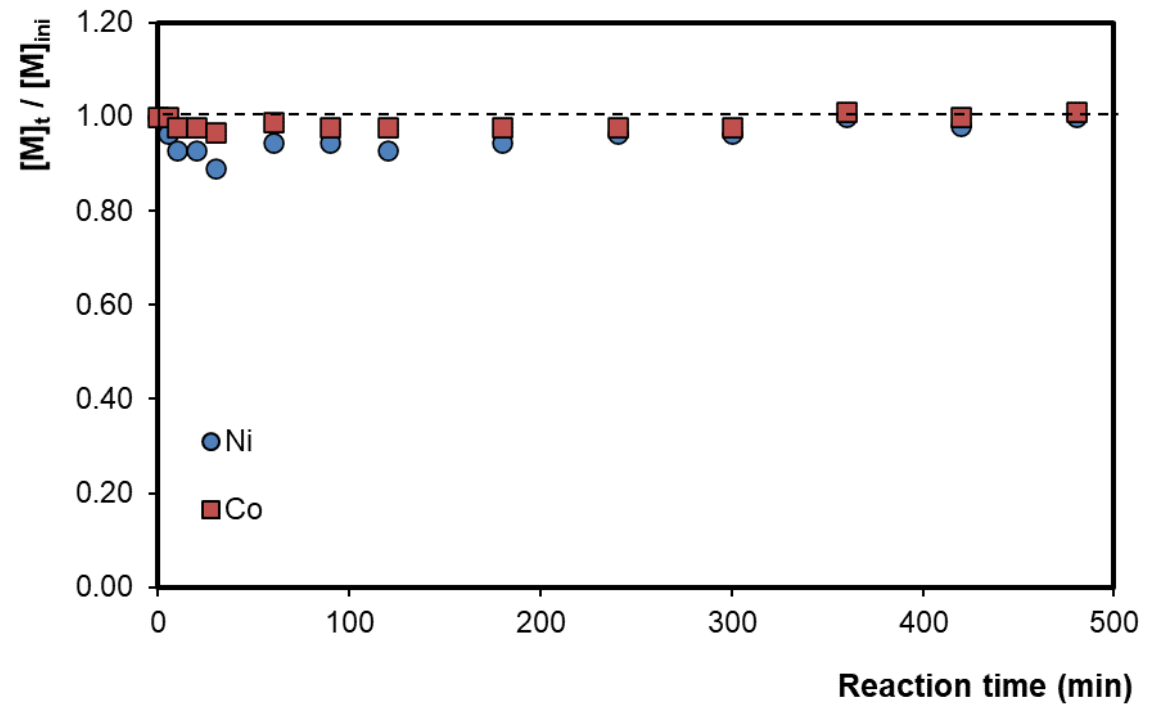

Figure S2. Evolution of the cobalt and nickel concentration with time during the continued ozonation of manganese in $3.7 \mathrm{wt} . \% \mathrm{HCl}$ solution. The concentration of cobalt and nickel are standardized according to their initial concentration (Mn:Co:Ni molar ratio of $0.07: 0.17: 1.00$ and $\left[\mathrm{NiCl}_{2} \cdot 6 \mathrm{H}_{2} \mathrm{O}\right]_{\text {ini }}=0.32 \mathrm{~mol} \cdot \mathrm{kg}^{-}$ $\left.{ }^{1}\right)$. 


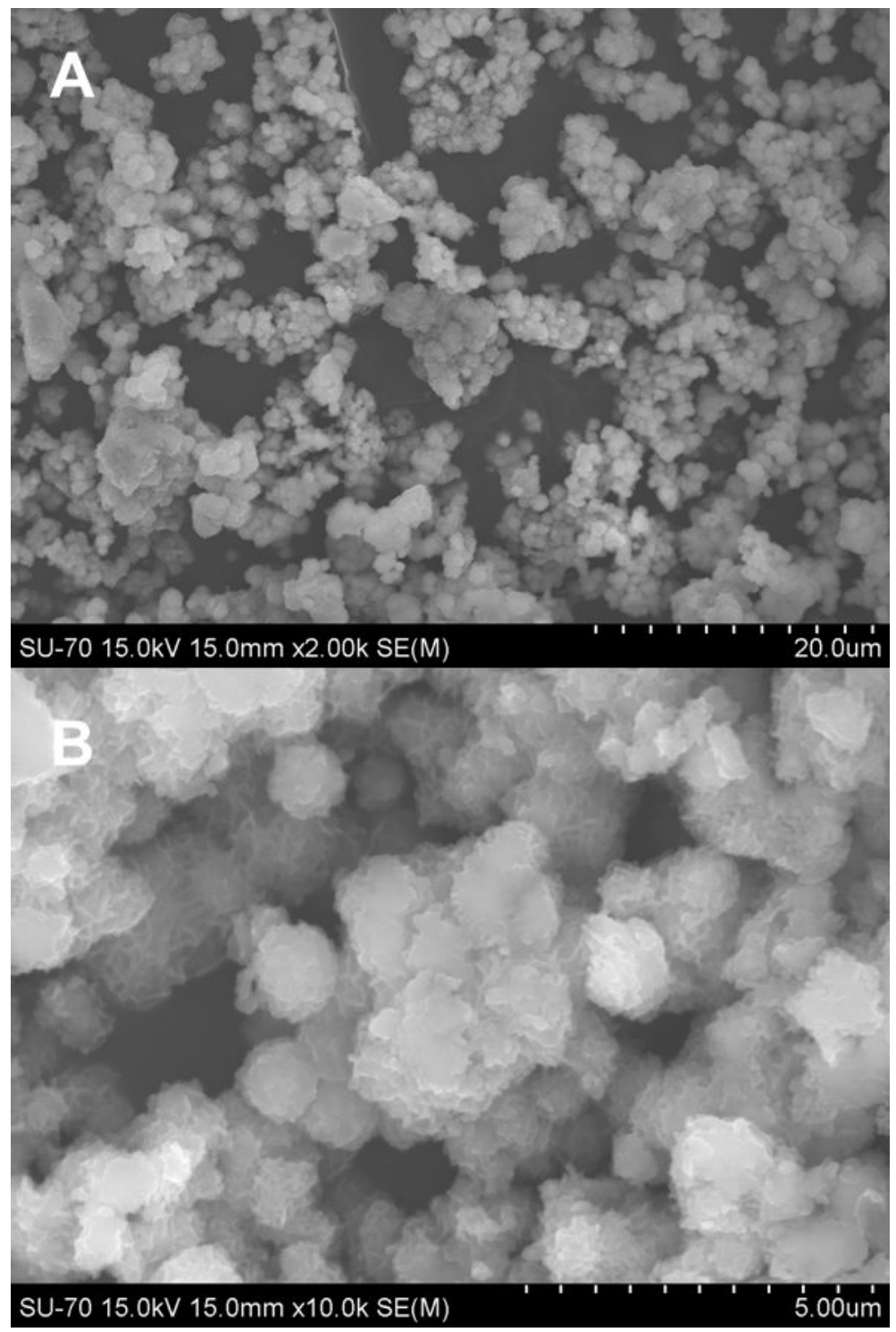

Figure S3. SEM image of the precipitate recovered after $480 \mathrm{~min}$ ozonation from neutral aqueous solution under A) low and B) high magnification. 


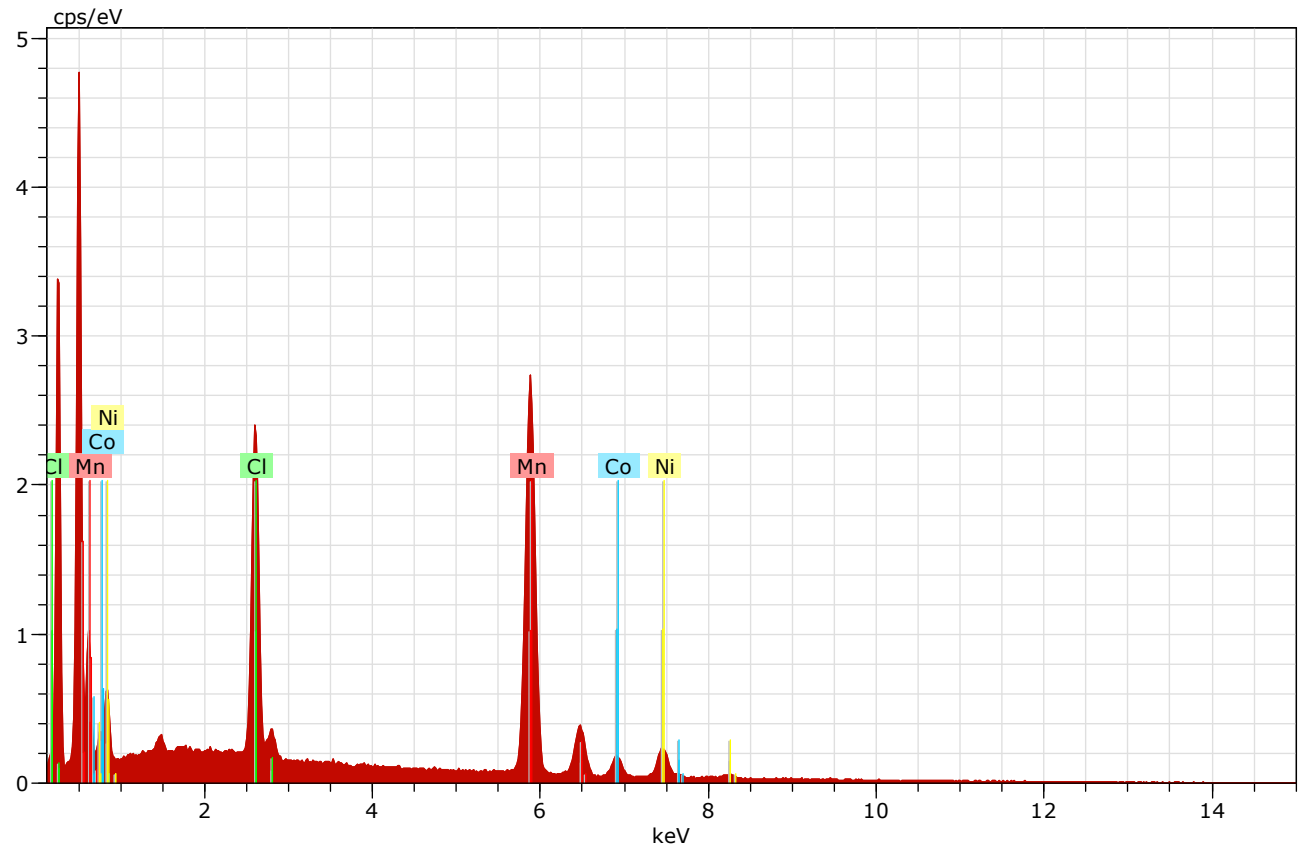

Figure S4. Energy dispersive X-ray analysis of the precipitate in Figure S3 recovered after 480 min from an aqueous solution.

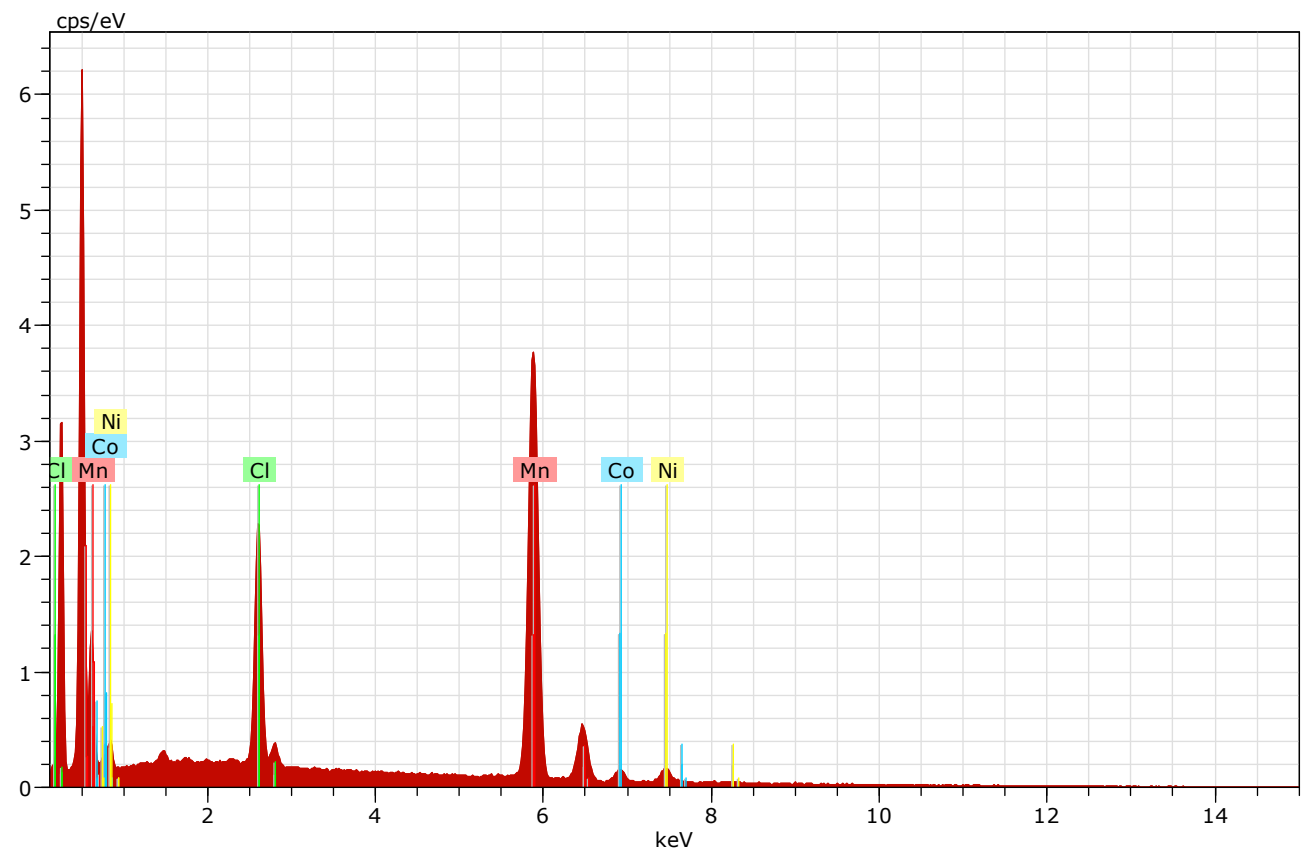

Figure S5. Energy dispersive X-ray analysis of the precipitate in Figure 2 of the manuscript recovered after $480 \mathrm{~min}$ from a $3.7 \mathrm{wt} . \% \mathrm{HCl}$ solution. 

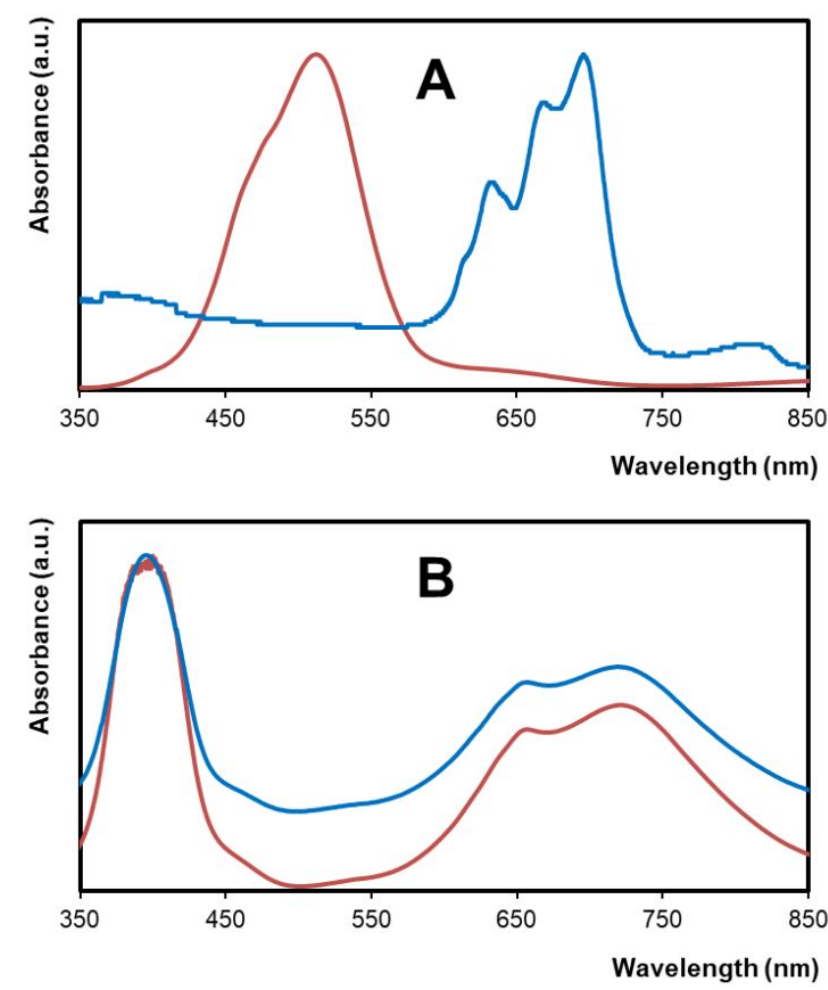

Figure S6. UV-vis spectra of the IL-rich (blue) and salt-rich (red) phases in the A) $\mathrm{CoCl}_{2}-\left[\mathrm{P}_{44414}\right] \mathrm{Cl}_{-} \mathrm{H}_{2} \mathrm{O}$ $\mathrm{ABS}$ and $\mathrm{B})$ the $\mathrm{NiCl}_{2}-\left[\mathrm{P}_{44414}\right] \mathrm{Cl}-\mathrm{H}_{2} \mathrm{O}$ at $\mathrm{T}=50.0{ }^{\circ} \mathrm{C}\left(\left[\mathrm{P}_{44414}\right] \mathrm{Cl}=30.0\right.$ wt.\%, $\left[\mathrm{NiCl}_{2}\right]=\left[\mathrm{CoCl}_{2}\right]=10.0$ wt.\%).

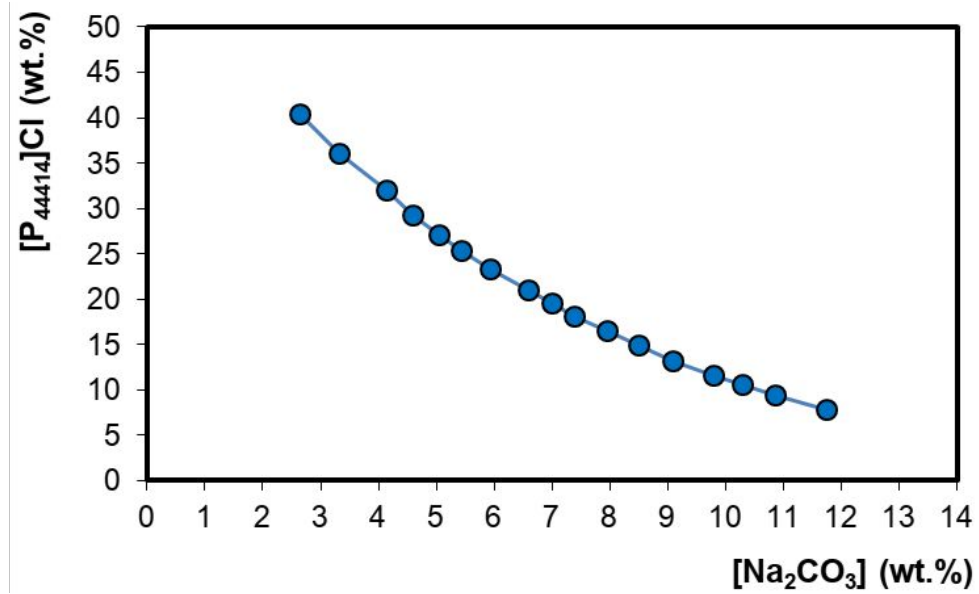

Figure S7. Binodal curves of the $\mathrm{Na}_{2} \mathrm{CO}_{3}-\left[\mathrm{P}_{4444}\right] \mathrm{Cl}-\mathrm{H}_{2} \mathrm{O}$ ABS at $25.0{ }^{\circ} \mathrm{C}$. 

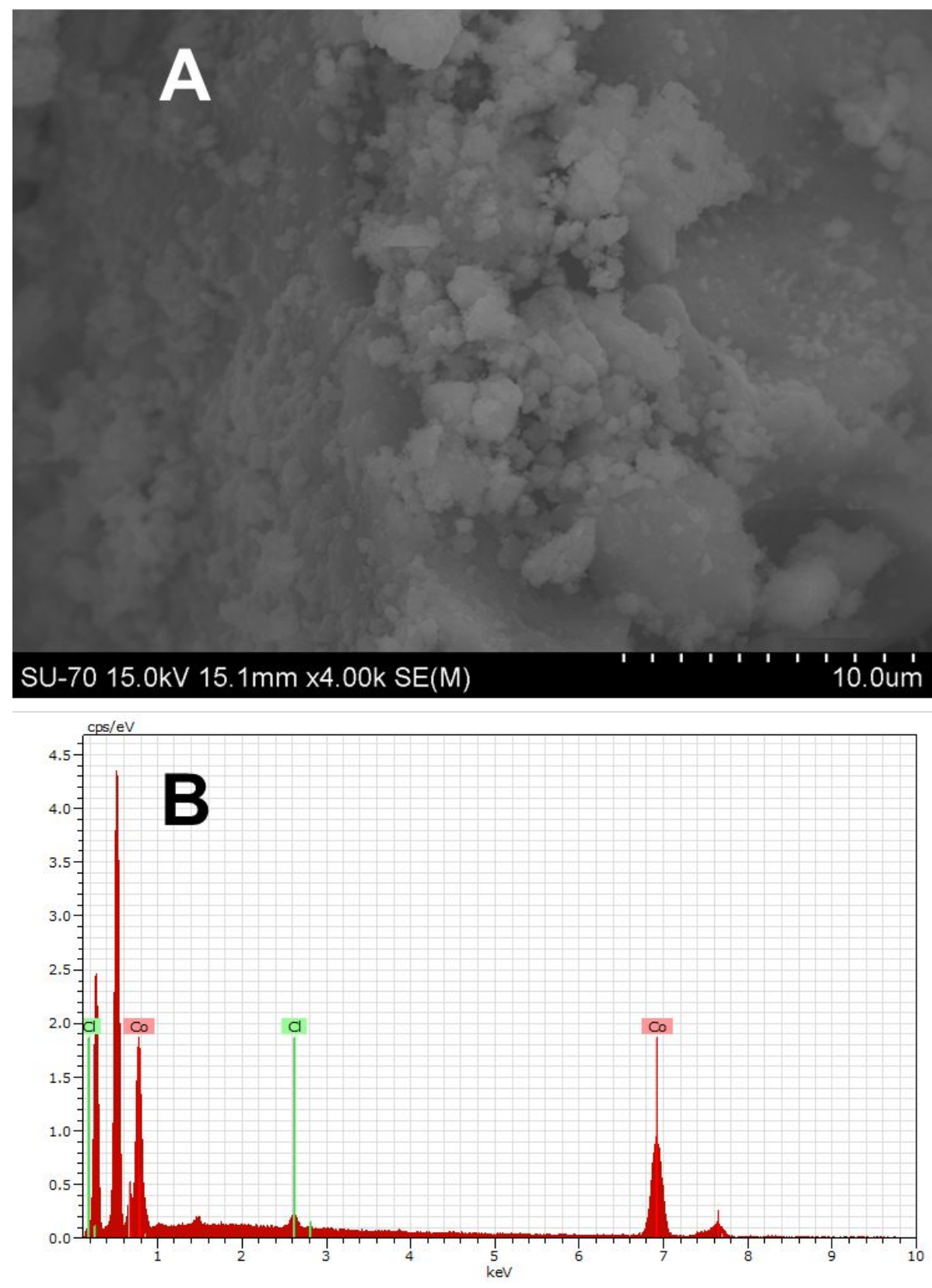

Figure S8. A) SEM image and B) Energy dispersive X-ray analysis of the recovered precipitate obtained after $8 \mathrm{x}$ dilution of isolated IL phase and subsequent addition of 1.2 stochiometric amount $\mathrm{Na}_{2} \mathrm{CO}_{3}$ (extraction conditions $-\chi_{\mathrm{Co} / \mathrm{Ni}}=0.20,\left[\mathrm{P}_{44414}\right] \mathrm{Cl}=20.0 \mathrm{wt} . \%$ and $\left[\mathrm{NiCl}_{2} \cdot 6 \mathrm{H}_{2} \mathrm{O}\right]=1.00 \mathrm{~mol} \cdot \mathrm{kg}^{-1}$ ). 

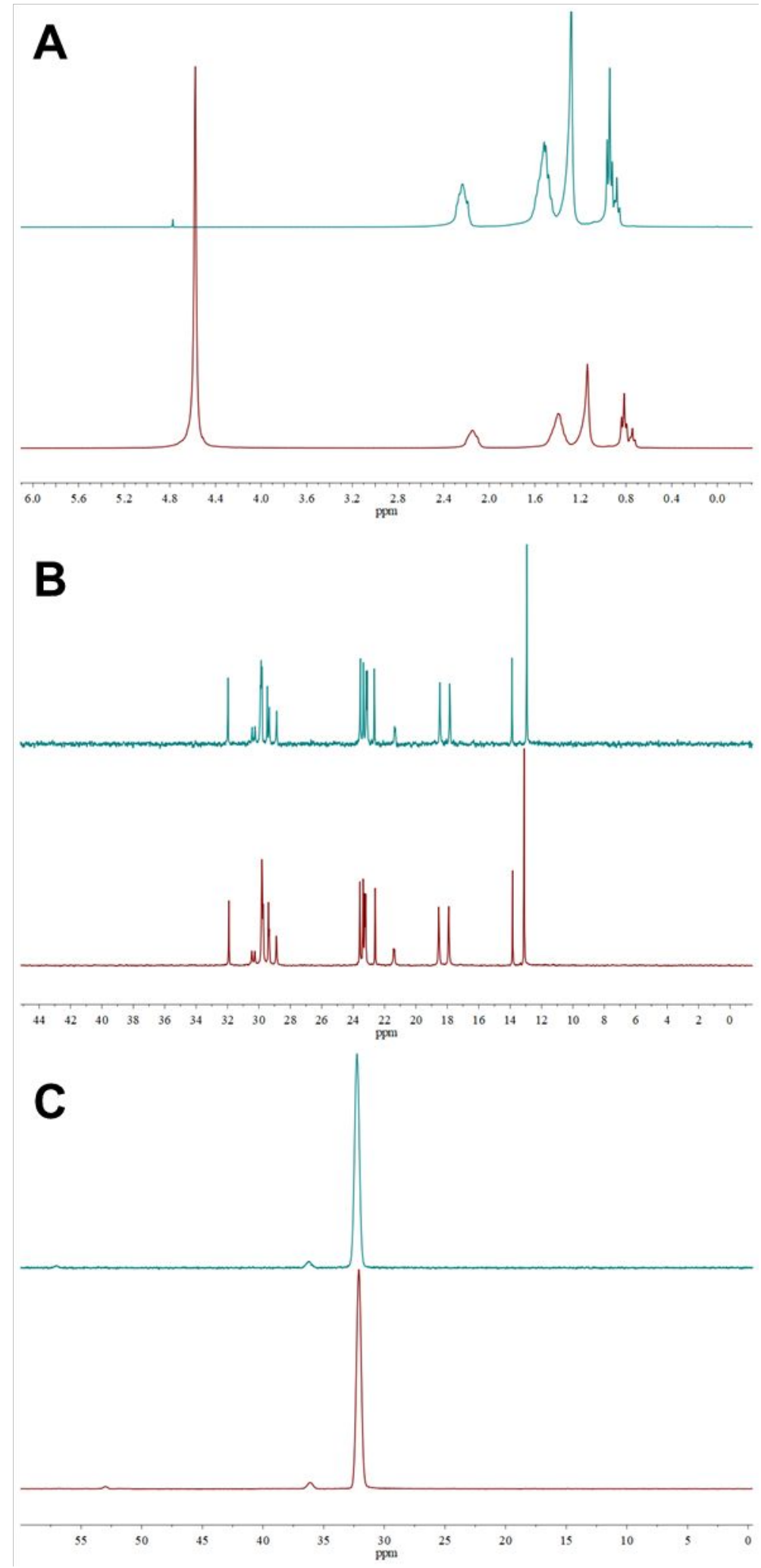

Figure S9. A) $\left.{ }^{1} \mathrm{H}-\mathrm{NMR}, \mathrm{B}\right){ }^{12} \mathrm{C}-\mathrm{NMR}$, and C) ${ }^{31} \mathrm{P}-\mathrm{NMR}$ spectra of virgin (blue) and recovered (red) $\left[\mathrm{P}_{44414}\right] \mathrm{Cl}$ (solvent $-\mathrm{D}_{2} \mathrm{O}$ ). 


\section{TABLES}

Table S1. Experimental binodal data for the ternary and quaternary systems reported in this work.

\begin{tabular}{|c|c|c|c|c|c|c|c|}
\hline \multicolumn{2}{|c|}{$25.0^{\circ} \mathrm{C}$} & \multicolumn{2}{|c|}{$50.0^{\circ} \mathrm{C}$} & \multicolumn{2}{|c|}{$50.0^{\circ} \mathrm{C}+3.7 \mathrm{wt} . \% \mathrm{HCl}$} & \multicolumn{2}{|c|}{$25.0^{\circ} \mathrm{C}$} \\
\hline$\left[\mathrm{NiCl}_{2}\right]$ & {$\left[\mathrm{P}_{44414}\right] \mathrm{Cl}$} & {$\left[\mathrm{NiCl}_{2}\right]$} & {$\left[\mathrm{P}_{44414}\right] \mathrm{Cl}$} & {$\left[\mathrm{NiCl}_{2}\right]$} & {$\left[\mathrm{P}_{44414}\right] \mathrm{Cl}$} & {$\left[\mathrm{Na}_{2} \mathrm{CO}_{3}\right]$} & {$\left[\mathbf{P}_{44414}\right] \mathrm{Cl}$} \\
\hline 6.51 & 71.02 & 2.47 & 56.80 & 0.93 & 56.71 & 2.64 & 40.33 \\
\hline 7.63 & 64.04 & 2.55 & 46.91 & 0.93 & 53.17 & 3.32 & 36.00 \\
\hline 7.75 & 60.06 & 2.55 & 45.12 & 0.94 & 49.51 & 4.15 & 31.92 \\
\hline 8.35 & 57.62 & 2.57 & 43.67 & 0.95 & 45.75 & 4.59 & 29.17 \\
\hline 8.46 & 55.51 & 2.64 & 42.39 & 0.94 & 42.65 & 5.06 & 27.08 \\
\hline 8.99 & 51.67 & 2.69 & 40.82 & 1.01 & 38.63 & 5.44 & 25.32 \\
\hline 9.63 & 48.12 & 2.74 & 39.81 & 1.02 & 35.04 & 5.93 & 23.35 \\
\hline 9.86 & 46.62 & 2.77 & 38.73 & 1.04 & 32.20 & 6.59 & 20.95 \\
\hline 10.26 & 44.92 & 2.86 & 37.62 & 1.11 & 28.10 & 7.00 & 19.49 \\
\hline 10.80 & 40.76 & 2.88 & 36.74 & 1.10 & 24.10 & 7.39 & 18.15 \\
\hline 11.58 & 36.80 & 2.91 & 35.60 & 1.16 & 21.32 & 7.96 & 16.49 \\
\hline 11.71 & 35.90 & 2.94 & 34.85 & 1.24 & 19.47 & 8.51 & 14.87 \\
\hline 11.96 & 34.25 & 2.99 & 33.95 & 1.25 & 18.63 & 9.10 & 13.24 \\
\hline 12.05 & 33.01 & 3.05 & 32.88 & 1.29 & 17.52 & 9.80 & 11.58 \\
\hline 12.17 & 32.25 & 3.04 & 31.73 & 1.33 & 16.50 & 10.29 & 10.50 \\
\hline 12.22 & 31.62 & 3.14 & 30.96 & 1.39 & 15.72 & 10.86 & 9.35 \\
\hline 12.45 & 30.72 & 3.21 & 30.04 & 1.46 & 14.52 & 11.76 & 7.76 \\
\hline 12.57 & 29.57 & 3.29 & 29.02 & 1.51 & 13.58 & & \\
\hline 12.90 & 28.12 & 3.29 & 28.37 & 1.58 & 12.85 & & \\
\hline 13.13 & 26.88 & 3.40 & 27.19 & 1.64 & 11.95 & & \\
\hline 13.37 & 25.80 & 3.42 & 26.40 & 1.71 & 11.16 & & \\
\hline 13.43 & 25.01 & 3.47 & 25.64 & 1.78 & 10.49 & & \\
\hline 13.49 & 24.19 & 3.50 & 24.85 & 1.87 & 9.56 & & \\
\hline 13.76 & 23.16 & 3.63 & 23.81 & 1.91 & 9.05 & & \\
\hline 13.80 & 22.53 & 3.68 & 22.76 & 2.04 & 8.17 & & \\
\hline 14.00 & 21.51 & 3.76 & 21.90 & 2.06 & 7.63 & & \\
\hline 14.27 & 20.18 & 3.82 & 21.05 & 2.09 & 7.28 & & \\
\hline 14.31 & 19.31 & 3.86 & 20.36 & 2.18 & 6.80 & & \\
\hline 14.34 & 18.87 & 3.93 & 19.63 & 2.20 & 6.62 & & \\
\hline 14.43 & 18.38 & 3.98 & 19.03 & 2.25 & 6.22 & & \\
\hline 14.49 & 17.92 & 4.13 & 17.94 & 2.40 & 5.12 & & \\
\hline 14.60 & 17.38 & 4.23 & 17.09 & & & & \\
\hline 14.80 & 16.49 & 4.36 & 16.54 & & & & \\
\hline 14.92 & 15.73 & 4.35 & 15.79 & & & & \\
\hline 15.04 & 15.03 & 4.46 & 14.65 & & & & \\
\hline
\end{tabular}




\begin{tabular}{|ll|ll|l|}
15.10 & 14.34 & 4.56 & 14.08 \\
15.29 & 13.67 & 4.74 & 12.57 \\
15.37 & 12.88 & 5.14 & 9.65 \\
15.54 & 12.15 & 5.31 & 8.40 \\
15.58 & 11.70 & 5.54 & 6.18 \\
15.65 & 11.24 & 5.81 & 4.51 & \\
15.74 & 10.82 & & \\
15.85 & 10.45 & & \\
15.89 & 10.12 & & \\
15.93 & 9.88 & & \\
16.04 & 9.58 & & \\
16.07 & 9.32 & & \\
16.10 & 9.07 & & \\
16.19 & 8.79 & & \\
16.21 & 8.71 & & \\
16.18 & 8.63 & & & \\
16.22 & 8.50 & & & \\
16.26 & 8.38 & & & \\
16.28 & 8.28 & & & \\
16.35 & 8.10 & & & \\
17.09 & 4.60 & & & \\
\end{tabular}


Table S2. Cobalt and nickel distribution coefficients $\left(D_{M}\right)$ as a function of the cobalt:nickel molar ratio $\left(\chi_{\mathrm{Co} / \mathrm{Ni}}\right)$ presented in Figure 5A. Distribution coefficients and cobalt-nickel separation factor $\left(\alpha_{\mathrm{Co} / \mathrm{Ni}}\right)$ are the average value from three separate measurements. Extraction conditions - $\left[\mathrm{P}_{44414}\right] \mathrm{Cl}=20.0$ wt.\%, $\left[\mathrm{NiCl}_{2} \cdot 6 \mathrm{H}_{2} \mathrm{O}\right]=0.35 \mathrm{~mol} . \mathrm{kg}^{-1},[\mathrm{HCl}]=1.00 \mathrm{~mol} . \mathrm{kg}^{-1}$.

\begin{tabular}{ccccccc}
\hline \multirow{2}{*}{$\chi_{\mathrm{Co} / \mathrm{Ni}}$} & \multicolumn{2}{c}{$\mathrm{D}_{\mathrm{Ni}}$} & \multicolumn{2}{c}{$\mathrm{D}_{\mathrm{Co}}$} & \multicolumn{2}{c}{$\alpha_{\mathrm{Co} / \mathrm{Ni}}$} \\
\cline { 2 - 7 } & Average & $\mathrm{STD}$ & Average & $\mathrm{STD}$ & Average & $\mathrm{STD}$ \\
\hline 0.00 & 0.21 & 0.02 & 0.00 & 0.00 & 0.00 & 0.00 \\
0.05 & 0.20 & 0.01 & 0.47 & 0.11 & 2.36 & 0.71 \\
0.10 & 0.16 & 0.01 & 0.62 & 0.13 & 3.98 & 0.94 \\
0.15 & 0.15 & 0.01 & 0.75 & 0.11 & 5.07 & 0.75 \\
0.20 & 0.12 & 0.01 & 0.79 & 0.03 & 6.72 & 0.59 \\
0.25 & 0.12 & 0.01 & 0.96 & 0.04 & 8.36 & 0.93 \\
0.33 & 0.10 & 0.02 & 1.43 & 0.04 & 14.9 & 2.74 \\
\hline
\end{tabular}

Table S3. Cobalt and nickel distribution coefficients $\left(D_{M}\right)$ as a function of the cobalt:nickel molar ratio $\left(\chi_{\mathrm{Co} / \mathrm{Ni}}\right)$ presented in Figure 5B. Distribution coefficients and cobalt-nickel separation factor $\left(\alpha_{\mathrm{Co} / \mathrm{Ni}}\right)$ are the average value from three separate measurements. Extraction conditions - $\left[\mathrm{P}_{44414}\right] \mathrm{Cl}=20.0 \mathrm{wt} . \%$, $\left[\mathrm{NiCl}_{2} \cdot 6 \mathrm{H}_{2} \mathrm{O}\right]=1.00 \mathrm{~mol} \cdot \mathrm{kg}^{-1},[\mathrm{HCl}]=0.00 \mathrm{~mol} . \mathrm{kg}^{-1}$.

\begin{tabular}{ccccccc}
\hline \multirow{2}{*}{$\chi_{\mathrm{Co} / \mathrm{Ni}}$} & \multicolumn{2}{c}{$\mathrm{D}_{\mathrm{Ni}}$} & \multicolumn{2}{c}{$\mathrm{D}_{\mathrm{Co}}$} & \multicolumn{2}{c}{$\alpha_{\mathrm{Co} / \mathrm{Ni}}$} \\
\cline { 2 - 7 } & Average & STD & Average & STD & Average & STD \\
\hline 0.00 & 0.10 & 0.01 & 0.00 & 0.00 & 0.00 & 0.00 \\
0.05 & 0.09 & 0.02 & 3.23 & 0.08 & 39.1 & 11.8 \\
0.10 & 0.07 & 0.02 & 4.45 & 0.11 & 71.3 & 21.8 \\
0.15 & 0.06 & $>0.01$ & 6.29 & 0.29 & 114.9 & 10.4 \\
0.20 & 0.03 & 0.01 & 12.2 & 1.50 & 483.9 & 77.7 \\
0.25 & 0.02 & $>0.01$ & 5.28 & 0.67 & 366.4 & 122.8 \\
0.33 & 0.01 & $>0.01$ & 3.17 & 0.37 & 292.3 & 92.0 \\
\hline
\end{tabular}

